Research Article

\title{
Modular Irregular Labeling on Double-Star and Friendship Graphs
}

\author{
K. A. Sugeng $\left(1,{ }^{1}\right.$ Z. Z. Barack, ${ }^{1}$ N. Hinding $\mathbb{D}^{2},{ }^{2}$ and R. Simanjuntak $\oplus^{3}$ \\ ${ }^{1}$ Department of Mathematics, Faculty of Mathematics and Natural Sciences, Universitas Indonesia, Depok, Indonesia \\ ${ }^{2}$ Department of Mathematics, Faculty of Mathematics and Natural Sciences, University of Hasanuddin, Makassar, Indonesia \\ ${ }^{3}$ Combinatorial Mathematics Research Group, Faculty of Mathematics and Natural, Sciences, Institute Technology Bandung, \\ Bandung, Indonesia \\ Correspondence should be addressed to K. A. Sugeng; kiki@sci.ui.ac.id
}

Received 17 September 2021; Revised 8 October 2021; Accepted 23 November 2021; Published 28 December 2021

Academic Editor: Ali Jaballah

Copyright $\odot 2021 \mathrm{~K}$. A. Sugeng et al. This is an open access article distributed under the Creative Commons Attribution License, which permits unrestricted use, distribution, and reproduction in any medium, provided the original work is properly cited.

\begin{abstract}
A modular irregular graph is a graph that admits a modular irregular labeling. A modular irregular labeling of a graph $G$ of order $n$ is a mapping of the set of edges of the graph to $\{1,2, \ldots, k\}$ such that the weights of all vertices are different. The vertex weight is the sum of its incident edge labels, and all vertex weights are calculated with the sum modulo $n$. The modular irregularity strength is the minimum largest edge label such that a modular irregular labeling can be done. In this paper, we construct a modular irregular labeling of two classes of graphs that are biregular; in this case, the regular double-star graph and friendship graph classes are chosen. Since the modular irregularity strength of the friendship graph also holds the minimal irregularity strength, then the labeling is also an irregular labeling with the same strength as the modular case.
\end{abstract}

\section{Introduction}

Graph labeling is a mapping of a set of numbers, called the labels, to the graph elements, usually vertices or edges [1]. Generally, the label is a positive integer. There are several labelings that have been developed; among them are irregular labeling and modular irregular labeling. The reader can check the dynamic survey of graph labeling by Gallian to obtain more information on various labeling [1]. In 1988, irregular labeling was first introduced by Chartrand et al. [2] . To date, there have been studies on the irregular labelings of certain graphs. The terminology not included in this paper can be found in [3].

An irregular labeling is defined as a labeling $f: E \longrightarrow\{1,2, \ldots, k\}$ with $k$ as a positive integer, such that $w t_{f(x)}=\sum_{(y \in N(x))} f(x y)$ is different for all vertices, where $N(x)$ is a neighbour of vertex $x$. The irregularity strength $s(G)$ of a graph $G$ is the minimum value of $k$ for which $G$ has irregular labeling with labels at most $k$. The irregularity strength $s(G)$ of a graph $G$ is defined only for graphs containing at most one isolated vertex and no connected component of order 2 . The lower bound of the irregularity strength of a graph $G$ is $s(G) \geq \max _{1 \leq i \leq \Delta}\left\{n_{i}+i-1 / i\right\}$, where $n_{i}$ vertices with degree $i$, as stated in Theorem 1 . For a regular graph $G$, Przyboylo [4] has proved an upper bound of an irregularity strength is $s(G)<16 n / d+6$. For tree graphs, Aigner and Triesch [5] proved that the irregularity strength of any tree with no vertices of degree two is equal to the number of its leaves. Ferrara et al. [6] later proved that if the tree $T$ has every two vertices of degree not equal to two at a distance of at least eight with number of leaves at least three, then $s(T)=n_{1}+n_{2} / 2$, where $n_{1}$ is the number of leaves and $n_{2}$ is the number of vertices of degree two. The survey of irregular labeling has been done by Bača et al. [7]. After this survey paper, there are still many results which have been found. See Gallian's survey, for the update [1].

Modular irregular labeling of a graph is a mapping $\varphi: E(G) \longrightarrow\{1,2, \ldots, k\}$ so that a bijective function $w t_{\varphi(x)}=\sum_{(y \in N(x))} \varphi(x y)$ can be defined and has different values. The set of the weights of the vertices is a group of integers modulo $n$. The minimum $k$ such that this kind of labeling exists is called the modular irregularity strength of $G$ 
and denoted by $m s(G)$. Bača et al. [8] determined the modular irregularity strength of path, star, triangular graph, cycle, and gear graphs. Muthugurupackiam et al. [9] proved the modular irregularity of the tadpole graph and doublecycle graph. Later, Bača et al. [10] proved the modular irregularity strength of the fan graph. In this paper, we construct the modular irregular labeling and determine its modular irregularity strength of regular double-star graph and friendship graph.

\section{Known Results}

There are some known results that we will use to prove the modular irregularity strength of the star and friendship graphs that we gave in this section. A lower bound on the irregularity strength is already known by Chartrand et al. as stated in the following theorem.

Theorem 1 (see [2]). Let $G$ be a connected graph with an order more than 2 , which has $n_{i}$ vertices with degree $i$. Then,

$$
s(G) \geq \max _{1 \leq i \leq \Delta(G)}\left\{\frac{n_{i}-1}{i}+1\right\} .
$$

The relation between the irregularity strength and modular irregularity strength has been known and presented in the following theorem.

Theorem 2 (see [8]). Let $G$ be a graph without a component of order $\leq 2$. Then,

$$
s(G) \leq m s(G)
$$

Not all graphs can have modular irregular labeling. In the following theorem, Bača et al. give a requirement of a graph that cannot have a modular irregular labeling, denoted by $m s(G)=\infty$.

Theorem 3 (see [8]). If $G$ is a graph of order $n$, $n \equiv 2(\bmod 4)$, then $G$ has no modular irregular $k$-labeling, i.e., $m s(G)=\infty$.

\section{New Results}

This section gives two results on a modular irregular labeling on a regular double-star graph and a friendship graph. Aman and Togni [5] and Ferrara et al. [6] proved the irregularity strength of trees family. The modular irregularity strength of the family of trees which is already known is path and star [8]. Since we consider biregular graphs in this paper, then the regular trees family that we choose is regular doublestars.

A regular double-star graph $S_{k, k}$ is a graph built from two copies of a star graph $S_{k}$, and then, we connect the two center vertices of the star. Note that a star $S_{k}$ has $k+1$ vertices. Thus, $S_{k, k}$ has $2 k+2$ vertices and $2 k+1$ edges.

Theorem 4. Let $S_{k, k}, k \geq 1$ be a regular double-star graph. Then,

$$
m s\left(S_{k, k}\right)= \begin{cases}2 k, & k \text { is odd } \\ \infty, & k \text { is even }\end{cases}
$$

Proof. Let $x$ and $y$ be the two centers of the double-star graph. Let $x_{i}, i=1, \ldots, k$, be the leaves of the center vertex $x$ and $y_{i}, i=1, \ldots, k$, be the leaves of the center vertex $y$.

For $k$ even, $\left|V\left(S_{k, k}\right)\right|=2 k+2 \equiv 2(\bmod 4)$. Then, following Theorem 3, the graph does not have a modular irregular labeling.

For $k$ odd, label the edges as follows.

For $1 \leq i \leq k$,

$$
\begin{aligned}
& \varphi\left(x x_{i}\right)= \begin{cases}2 i-1, & i \text { odd }, \\
2 i, & i \text { even, }\end{cases} \\
& \varphi\left(y y_{i}\right)= \begin{cases}2 i, & i \text { odd, } \\
2 i-1, & i \text { even, }\end{cases} \\
& \varphi(x y)=\frac{3 k+1}{2}<2 k .
\end{aligned}
$$

The pendant leaves should have the different labels; then, the minimal number of labels is $2 k$. The maximal label is at $\varphi\left(y y_{k}\right)=2 k$. Thus, $\varphi$ is a $2 k$-labeling. Its leaves weight will be elements of $\{1,2, \ldots, 2 k\}, w t_{\varphi}(x) \equiv 2 k+1(\bmod (2 k+2))$ and $w t_{\varphi}(y) \equiv 0(\bmod (2 k+2))$. All vertex weights are different. Thus, $m s\left(S_{k, k}\right)=2 k$ for $k$ odd.

A friendship graph $F_{n}$ is a graph with the vertex set $\left\{m, x_{1}, \ldots, x_{n}, y_{1}, \ldots, y_{n}\right\}$ and the edge set is $\left\{m x_{1}, \ldots\right.$, $\left.m x_{n}, m y_{1}, \ldots m y_{n}, x_{1} y_{1}, \ldots, x_{n} y_{n}\right\}$. Thus, the graph $F_{n}$ has $2 n+1$ vertices and $3 n$ edges. Since the graph has $|V(G)| \neq 2(\bmod 4)$, then based on Theorem 3 , we have a possibility to find the modular irregularity strength of $F_{n}$. In the following lemma, we have a lower bound of $m s\left(F_{n}\right)$.

Lemma 1. Let $F_{n}$ be a friendship graph with $n \geq 2$. Then,

$$
m s\left(F_{n}\right) \geq n+1 \text {. }
$$

Proof. A friendship graph $F_{n}$ has $2 n$ vertices with degree two and one vertex with degree $2 n$. Based on Theorem 1, we have

$$
\begin{aligned}
& s\left(F_{n}\right) \geq \max _{1 \leq i \leq 2 n}\left\{\frac{2 n-1}{2}+1, \frac{1-1}{2 n}+1\right\}, \\
& s\left(F_{n}\right) \geq n+\frac{1}{2}, \\
& s\left(F_{n}\right) \geq n+1\left(\text { since } s\left(F_{n}\right) \text { is an integer }\right) .
\end{aligned}
$$

Then, based on Theorem 2, we obtain

$$
\begin{aligned}
& m s\left(F_{n}\right) \geq s\left(F_{n}\right) \geq n+1 . \\
& m s\left(F_{n}\right) \geq n+1 .
\end{aligned}
$$

A modular irregular labeling can be constructed and $m s\left(F_{n}\right)$ can be determined, and the conclusion is written in the following theorem. 
Theorem 5. Let $F_{n}$ be a friendship graph with $n \geq 2$. Then, $m s$ $\left(F_{n}\right)=n+1$.

Proof. We divide the proof in 4 cases. In each case, we define the edge labeling $\varphi: E(G) \longrightarrow\{1,2, \ldots, 3 n\}$ and show that $\phi$ is an $(n+1)$-labeling. Then, in the second step, we show that the vertex weights are all different.

\subsection{Case $n \equiv 0(\bmod 4)$}

Label the edges as follows.

$$
\begin{aligned}
& \varphi\left(x_{i} y_{i}\right)= \begin{cases}2, & i=1, \\
i+2, & i=2,3, \ldots, \frac{n}{2} \text { and } i>\frac{n}{2}, i \text { odd }, \\
i+1, & i>\frac{n}{2}, i \text { even, }\end{cases} \\
& \varphi\left(x_{i} m\right)= \begin{cases}1, & i=1, \\
i-1, & i=2,3, \ldots, \frac{n}{2} \text { and } i>\frac{n}{2}, i \text { odd } \\
i, & i>\frac{n}{2}, i \text { even, }\end{cases} \\
& \varphi\left(y_{i} m\right)= \begin{cases}2, & i=1, \\
i, & i=2,3, \ldots, \frac{n}{2} \text { and } i>\frac{n}{2}, i \text { odd }\end{cases} \\
& i+1, \quad i>\frac{n}{2}, i \text { even. }
\end{aligned}
$$

(a) Let $\varphi$ be an edge labeling of the friendship graph $F_{n}$ that is defined above; we can obtain $\max \left\{\varphi\left(x_{i} y_{i}\right), \varphi\left(x_{i} m\right), \varphi\left(y_{i m}\right): 1 \leq i \leq n\right\}=n+1$.

Then, it is proved that the edge labeling $\varphi$ is an $(n+1)$-labeling.

(b) The edges adjacent to $x_{i}$ are $x_{i} y_{i}$ and $x_{i} m$ so that for $i=1$

$$
w t_{\varphi}\left(x_{1}\right)=\varphi\left(x_{1} y_{1}\right)+\varphi\left(x_{1} m\right)=2+1=3 \text {, }
$$

for $i=2,3, \ldots, n / 2$ and $i>n / 2, i$ odd,

$$
w t_{\varphi}\left(x_{i}\right)=\varphi\left(x_{i} y_{i}\right)+\varphi\left(x_{i} m\right)=2 i+1,
$$

for $i>n / 2, i$ even,

$$
w t_{\varphi}\left(x_{i}\right)=\varphi\left(x_{i} y_{i}\right)+\varphi\left(x_{i} m\right)=2 i+1 \text {. }
$$

Thus, we have the vertex weight, $w t_{\varphi}\left(x_{i}\right)=$ $2 i+1, i=1,2, \ldots, n$.

(c) The edges adjacent to $y_{i}$ is $x_{i} y_{i}$ and $y_{i} m$ so that for $i=1$,

$$
w t_{\varphi}\left(y_{1}\right)=\varphi\left(x_{1} y_{1}\right)+\varphi\left(y_{1} m\right)=2+2=4 \text {. }
$$

For $i=2,3, \ldots, n / 2$ and $i>n / 2, i$ odd,

$$
\begin{aligned}
w t_{\varphi}\left(y_{i}\right) & =\varphi\left(x_{i} y_{i}\right)+\varphi\left(y_{i} m\right) \\
& =i+2+i=2 \mathrm{i}+2 .
\end{aligned}
$$

For $i>n / 2, i$ even,

$$
\begin{aligned}
w t_{\varphi}\left(y_{i}\right) & =\varphi\left(x_{i} y_{i}\right)+\varphi\left(y_{i} m\right) \\
& =i+1+i+1=2 i+2 .
\end{aligned}
$$

Thus, we have the vertex weight, $w t_{\varphi}\left(y_{i}\right)=$ $2 i+2, i=1,2, \ldots, n$.

(d) The edges adjacent to $m$ are $x_{i} m$ and $y_{i} m$ so that

$$
\begin{aligned}
w t_{\varphi}(m)= & \sum_{i=1}^{n}\left(\varphi\left(x_{i} m\right)+\varphi\left(y_{i} m\right)\right) \\
= & 1+\sum_{i=2}^{\frac{n}{2}}(i-1)+\sum_{i=1}^{\frac{n}{4}}\left(\frac{n}{2}+(2 i-1)-1\right)+\sum_{i=1}^{\frac{4}{2}}\left(\frac{n}{2}+2 i\right) \\
& +2+\frac{n}{\frac{n}{2}} i+\frac{n}{4}\left(\frac{n}{2}+(2 i-1)\right)+\sum_{i=1}^{\frac{4}{2}}\left(\frac{n}{2}+2 i+1\right) \\
= & n^{2}+\frac{n}{2}+2=(2 n+1) \frac{n}{2}+2 \equiv 2(\bmod (2 n+1)) .
\end{aligned}
$$

\subsection{Case $n \equiv 1(\bmod 4), n>1$}

Label the edges as follows.

$$
\begin{aligned}
& \varphi\left(x_{i} y_{i}\right)= \begin{cases}i+1, & i=1,2, \ldots, \frac{n+3}{2} \text { and } i>\frac{n+3}{2}, i \text { odd. } \\
i+2, & i>\frac{n+3}{2}, i \text { even. }\end{cases} \\
& \varphi\left(x_{i} m\right)= \begin{cases}i, & i=1,2, \ldots, \frac{n+3}{2} \text { and } i>\frac{n+3}{2}, i \text { odd. } \\
i-1, & i>\frac{n+3}{2}, i \text { even. }\end{cases} \\
& \varphi\left(y_{i} m\right)= \begin{cases}i+1, & i=1,2, \ldots, \frac{n+3}{2} \text { and } i>\frac{n+3}{2}, i \text { odd. } \\
i, & i>\frac{n+3}{2}, i \text { even. }\end{cases}
\end{aligned}
$$

(a) Let $\varphi$ be an edge labeling of the friendship graph $F_{n}$ that is defined above; we can have $\max$ $\left\{\varphi\left(x_{i} y_{i}\right), \varphi\left(x_{i} m\right), \varphi\left(y_{i m}\right): 1 \leq i \leq n=n+1\right.$. 
Then, it is proved that the edge labeling $\varphi$ is an $(n+1)$-labeling for the graph $F_{n}$.

(b) The edges adjacent to $x_{i}$ is $x_{i} y_{i}$ and $x_{i} m$ so that, for $i=1,2, \ldots, n+3 / 2$ and $i>n+3 / 2, i$ odd,

$$
\begin{aligned}
w t_{\varphi}\left(x_{i}\right) & =\varphi\left(x_{i} y_{i}\right)+\varphi\left(x_{i} m\right) \\
& =i+1+i=2 i+1 .
\end{aligned}
$$

For $i>n+3 / 2$, $i$ even,

$$
\begin{aligned}
w t_{\varphi}\left(x_{i}\right) & =\varphi\left(x_{i} y_{i}\right)+\varphi\left(x_{i} m\right) \\
& =i+2+i-1=2 i+1 .
\end{aligned}
$$

Then, we have the vertex weight, $w t_{\varphi}\left(x_{i}\right)=$ $2 i+1, i=1,2, \ldots, n$.

(c) The edges adjacent to $y_{i}$ are $x_{i} y_{i}$ and $y_{i} m$ so that, for $i=1,2, \ldots, n+3 / 2$ and $i>n+3 / 2, i$ odd,

$$
\begin{aligned}
w t_{\varphi}\left(y_{i}\right) & =\varphi\left(x_{i} y_{i}\right)+\varphi\left(y_{i} m\right) \\
& =i+1+i+1=2 i+2 .
\end{aligned}
$$

For $i>n+3 / 2, i$ even,

$$
\begin{aligned}
w t_{\varphi}\left(y_{i}\right) & =\varphi\left(x_{i} y_{i}\right)+\varphi\left(y_{i} m\right) \\
& =i+2+i=2 i+2 .
\end{aligned}
$$

Then, we obtain the vertex weight $w t_{\varphi}\left(y_{i}\right)=$ $2 i+2, i=1,2, \ldots, n$.

(d) The edges adjacent to $m$ are $x_{i} m$ and $y_{i} m$ so that

$$
\begin{aligned}
w t_{\varphi}(m)= & \sum_{i=1}^{n}\left(\varphi\left(x_{i} m\right)+\varphi\left(y_{i} m\right)\right) \\
& \frac{n+3}{\sum_{i=1}^{2}} i+\frac{n-1}{\sum_{i=1}^{4}}\left(\frac{n+3}{2}+(2 i-1)\right) \\
& \frac{n-5}{4}\left(\frac{n+3}{2}+2 i-1\right) \\
& +\frac{n+3}{i=1} \quad \frac{n-1}{4}\left(\frac{n+3}{2}+(2 i-1)+1\right) \\
& +\sum_{i=1}^{2}(i+1)+\sum_{i=1}^{n}\left(\frac{n-5}{4}\left(\frac{n+3}{2}+2 i\right)\right. \\
& +\sum_{i=1}^{4}(2 n+1) \frac{n+1}{2}+2 \equiv 2(\bmod (2 n+1)) . \\
= & (2 n)
\end{aligned}
$$

\subsection{Case $n \equiv 2(\bmod 4)$}

Label the edges as follows:

$$
\begin{aligned}
& \varphi\left(x_{i} y_{i}\right)= \begin{cases}i, & i=1,2, \ldots, \frac{n}{2}, \\
i+1 & i>\frac{n}{2}, i \text { even } \\
i+2 & i>\frac{n}{2}, i \text { odd },\end{cases} \\
& \varphi\left(x_{i} m\right)= \begin{cases}i, & i=1,2, \ldots, \frac{n}{2} \\
i-1 & i>\frac{n}{2}, i \text { even }, \\
i-2 & i>\frac{n}{2}, i \text { odd } .\end{cases}
\end{aligned}
$$

(a) Let $\varphi$ be an edge labeling of the friendship graph $F_{n}$ that is defined above; we have max $\left\{\varphi\left(x_{i} y_{i}\right), \varphi\left(x_{i} m\right), \varphi\left(y_{i m}\right): 1 \leq i \leq n=n+1\right.$.

Then, it is proved that the edge labeling $\varphi$ is an $(n+1)$-labeling for the graph $F_{n}$.

(b) The edges adjacent to $x_{i}$ are $x_{i} y_{i}$ and $x_{i} m$ so that, for $i=1,2, \ldots, n / 2$,

$$
\begin{aligned}
w t_{\varphi}\left(x_{i}\right) & =\varphi\left(x_{i} y_{i}\right)+\varphi\left(x_{i} m\right) \\
& =i+i=2 i .
\end{aligned}
$$

For $i>n / 2, i$ even,

$$
\begin{aligned}
w t_{\varphi}\left(x_{i}\right) & =\varphi\left(x_{i} y_{i}\right)+\varphi\left(x_{i} m\right) \\
& =i+1+i-1=2 i .
\end{aligned}
$$

For $i>n / 2$, $i$ odd,

$$
\begin{aligned}
w t_{\varphi}\left(x_{i}\right) & =\varphi\left(x_{i} y_{i}\right)+\varphi\left(x_{i} m\right) \\
& =i+2+i-2=2 i .
\end{aligned}
$$

Then, we obtain the vertex weight $w t_{\varphi}\left(x_{i}\right)=2 i$, $i=1,2, \ldots, n$.

(c) The edges adjacent to $y_{i}$ are $x_{i} y_{i}$ and $y_{i} m$ so that, for $i=1,2, \ldots, n / 2$,

$$
\begin{aligned}
w t_{\varphi}\left(y_{i}\right) & =\varphi\left(x_{i} y_{i}\right)+\varphi\left(y_{i} m\right) \\
& =i+i+1=2 i+1 .
\end{aligned}
$$

For $i>n / 2, i$ even,

$$
\begin{aligned}
w t_{\varphi}\left(y_{i}\right) & =\varphi\left(x_{i} y_{i}\right)+\varphi\left(y_{i} m\right) \\
& =i+1+i=2 i+1 .
\end{aligned}
$$

For $i>n / 2$, $i$ odd, 


$$
\begin{aligned}
w t_{\varphi}\left(y_{i}\right) & =\varphi\left(x_{i} y_{i}\right)+\varphi\left(y_{i} m\right) \\
& =i+2+i-1=2 i+1
\end{aligned}
$$

Then, we conclude that the vertex weight $w t_{\varphi}\left(y_{i}\right)=2 i+1, i=1,2, \ldots, n$.

(d) The edges adjacent to $m$ are $x_{i} m$ and $y_{i} m$ so that

$$
\begin{aligned}
w t_{\varphi}(m)= & \sum_{i=1}^{n}\left(\varphi\left(x_{i} m\right)+\varphi\left(y_{i} m\right)\right) \\
& \frac{n}{\frac{2}{2}} i+\frac{n+2}{\sum^{4}}\left(\frac{n}{2}+(2 i-1)-1\right)+\sum_{i=1}^{\frac{n-2}{4}}\left(\frac{n}{2}+2 i-2\right) \\
= & \sum_{i=1} i \frac{n+2}{4}\left(\frac{n}{2}+(2 i-1)\right)+\sum_{i=1}^{\frac{n}{2}}\left(\frac{n}{2}+2 i-1\right) \\
& +\sum_{i=1}^{\frac{n}{2}}(i+1)+\sum_{i=1}^{n}(2 n+1) \frac{n}{2}+1 \equiv 1(\bmod (2 n+1)) .
\end{aligned}
$$

\subsection{Case $n \equiv 3(\bmod 4), n>3$}

Label the edges as follows:

$$
\begin{aligned}
& \varphi\left(x_{i} y_{i}\right)= \begin{cases}i, & i=1,2, \ldots, \frac{n+3}{2} \text { and } i>\frac{n+3}{2}, i \text { even, } \\
i+1, & i>\frac{n+3}{2}, i \text { odd },\end{cases} \\
& \varphi\left(x_{i} m\right)= \begin{cases}i, & i=1,2, \ldots, \frac{n+3}{2} \text { and } i>\frac{n+3}{2}, i \text { even, } \\
i-1, & i>\frac{n+3}{2}, i \text { odd },\end{cases} \\
& \varphi\left(y_{i m}\right)= \begin{cases}i+1, & i=1,2, \ldots, \frac{n+3}{2} \text { and } i>\frac{n+3}{2}, i \text { even, } \\
i, & i>\frac{n+3}{2}, i \text { odd. }\end{cases}
\end{aligned}
$$

(a) Let $\varphi$ be an edge labeling of the friendship graph $F_{n}$ that is defined above; we have max $\left\{\varphi\left(x_{i} y_{i}\right), \varphi\left(x_{i} m\right), \varphi\left(y_{i m}\right): 1 \leq i \leq n=n+1\right.$.

Then, it is proved that the edge labeling $\varphi$ is an $(n+1)$-labeling for the graph $F_{n}$.

(b) The edges adjacent to $x_{i}$ are $x_{i} y_{i}$ and $x_{i} m$ so that, for $i=1,2, \ldots, n+3 / 2$ and $i>n+3 / 2$, $i$ even,

$$
\begin{aligned}
w t_{\varphi}\left(x_{i}\right) & =\varphi\left(x_{i} y_{i}\right)+\varphi\left(x_{i} m\right) \\
& =i+i=2 i .
\end{aligned}
$$

For $i>n+3 / 2$, $i$ odd,

$$
\begin{aligned}
w t_{\varphi}\left(x_{i}\right) & =\varphi\left(x_{i} y_{i}\right)+\varphi\left(x_{i} m\right) \\
& =i+1+i-1=2 i
\end{aligned}
$$

Then, we have the vertex weight $w t_{\varphi}\left(x_{i}\right)=2 i$, $i=1,2, \ldots, n$.

(c) The edges adjacent to $y_{i}$ are $x_{i} y_{i}$ and $y_{i} m$ so that, for $i=1,2, \ldots, n+3 / 2$ and $i>n+3 / 2, i$ even,

$$
\begin{aligned}
w t_{\varphi}\left(y_{i}\right) & =\varphi\left(x_{i} y_{i}\right)+\varphi\left(y_{i} m\right) \\
& =i+i+1=2 i+1 .
\end{aligned}
$$

For $i>n+3 / 2, i$ odd,

$$
\begin{aligned}
w t_{\varphi}\left(y_{i}\right) & =\varphi\left(x_{i} y_{i}\right)+\varphi\left(y_{i} m\right) \\
& =i+1+i=2 i+1 .
\end{aligned}
$$

Then, we can conclude that the vertex weight $w t_{\varphi}\left(y_{i}\right)=2 i+1, i=1,2, \ldots, n$.

(d) The edges adjacent to $m$ are $x_{i} m$ and $y_{i} m$ so that

$$
w t_{\varphi}(m)=\sum_{i=1}^{n}\left(\varphi\left(x_{i} m\right)+\varphi\left(y_{i} m\right)\right)
$$$$
\frac{n+3}{\sum_{i=1}^{2}} i+\frac{n-3}{\sum_{i=1}^{4}\left(\frac{n+3}{2}+(2 i-1)\right)}
$$$$
+\sum_{i=1}^{\frac{n-3}{4}}\left(\frac{n+3}{2}+2 i-1\right)
$$$$
+\sum_{i=1}^{\frac{n+3}{2}}(i+1)+\sum_{i=1}^{\frac{n-3}{4}}\left(\frac{n+3}{2}+(2 i-1)+1\right)
$$$$
+\sum_{i=1}^{\frac{n-3}{4}}\left(\frac{n+3}{2}+2 i\right)
$$

$$
w t_{\varphi}(m)=(2 n+1) \frac{n+1}{2}+1 \equiv 1 \bmod (2 k(2 n+1)) .
$$

In all cases, we proved that the vertex weights are all different and the maximum label is $n+1$. By combining the results $\left(m s\left(F_{n}\right) \leq n+1\right)$ and Lemma $1\left(m s\left(F_{n}\right) \geq n+1\right)$, we conclude that $m s\left(F_{n}\right)=n+1$, for $n \geq 2$.

From Theorem 5 , we have $m s\left(F_{n}\right)=n+1$ is equal to the lower bound of the irregularity strength from Theorem 1 . We can conclude that the friendship graph has irregular labeling with $s\left(F_{n}\right)=n+1$.

Corollary 1. The friendship graph $F_{n}$ has the irregularity strength $s\left(F_{n}\right)=n+1$ for $n \geq 2$. 


\section{Conclusion}

In this paper, we prove the modular irregularity strength of two graphs, which are the regular double-star graph $S_{k, k}$, that has $m s\left(S_{k, k}\right)=2 k$, for $k \geq 1$ and $k$ is odd and for the friendship graph $F_{n}$ that has $m s\left(F_{n}\right)=n+1$ and $s\left(F_{n}\right)=n+1$, for $n \geq 2$. There are still many families of graphs that can be explored to determine its modular irregularity strength.

\section{Data Availability}

No data were used to support this study.

\section{Conflicts of Interest}

The authors declare that there are no conflicts of interest.

\section{Authors' Contributions}

K.A.S. and Z.Z.B. conceptualized the study; K.A.S. developed the methodology; K.A.S., Z.Z.B, N.H., and R.S. validated the study; K.A.S. and Z.Z.B. wrote and prepared the original draft; K.A.S., Z.Z.B, N.H., and R.S. reviewed and edited the manuscript; K.A.S. helped with funding acquisition. All authors have read and agreed to the published version of the manuscript.

\section{Acknowledgments}

This research was funded by PPKI-UI Research (Grant no. NKB-461/UN2.RST/HKP.05.00/2021).

\section{References}

[1] J. A. Gallian, "A dynamic survey of graph labeling," The Electronic Journal of Combinatorics, vol. 19, 2020.

[2] G. Chartrand, M. S. Jacobon, J. Lehel, O. R. Oellermann, S. Ruiz, and F. Saba, "Irregular network," Congressus Numerantium, vol. 64, pp. 187-192, 1988.

[3] D. B. West, An Introduction to Graph Theory, Prentice-Hall, Hoboken, NY, USA, 1996.

[4] J. Przyboylo, "Irregularity strength of regular graphs," Electronic Journal of Combinatorics, vol. 15, 2008.

[5] M. Aigner and E. Triesch, "Irregular Assignments of Trees and Forests," SIAM Journal on Discrete Mathematics, vol. 3, no. 4, pp. 439-449, 1990.

[6] M. Ferrara, R. Gould, M. Pfender, and F. Pfender, "An iterative approach to graph irregularity strength," Discrete Applied Mathematics, vol. 158, no. 11, pp. 1189-1194, 2010.

[7] M. Bača, S. Jendrol, K. Kathiresan, K. Muthugurupackiam, and A. Semaničová-Feňovčíková, "A survey of irregularity strength," Electronic Notes in Discrete Mathematics, vol. 48, pp. 19-26, 2015.

[8] M. Baca, K. Muthugurupackiam, K. Kathiresan, and S. Ramya, "Modular irregularity strength of graphs," Electronic Journal of Graph Theory and Applications, vol. 8, no. 2, pp. 435-443, 2020.

[9] K. Muthugurupackiam and S. Ramya, "Modular irregularity strength of two classes of graphs," Journal of Computer and Mathematical Sciences, vol. 9, no. 9, pp. 1132-1141, 2018.
[10] M. Bača, Z. Kimákov’, M. Lascsáková, and A. SemaničováFeňovč́ková, "The irregularity and modular irregularity strength of fan graphs," Symmetry, vol. 13, no. 4, p. 605, 2021. 